\title{
Functional Approach without Path Integrals to Finite Temperature Free Fermions
}

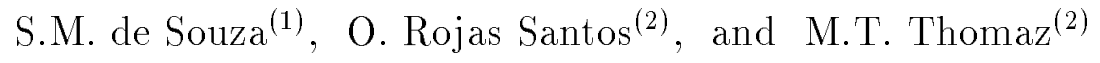 \\ (1) Departamento de Ciências Exatas \\ Universidade Federal de Lavras \\ Caixa Postal 37 \\ CEP: 37200-000, Lavras, MG, Brazil \\ (2) Instituto de Física \\ Universidade Federal Fluminense \\ Av. Gal. Milton Tavares de Souza $s / n$, \\ CEP: 24210-340, Niterói, RJ, Brazil
}

Received 30 June, 1999. Revised version received on 02 August, 1999

\begin{abstract}
Charret et al. applied the properties of Grassmann generators to develop a new method to calculate the coefficientes of the high temperature expansion of the grand canonical partition function of self-interacting fermionic models on $d$-dimensions $(d \geq 1)$. The method explores the anti-commuting nature of fermionic fields and avoids the calculation of the fermionic path integral. We apply this new method to the relativistic free Dirac fermions and recover the known results in the literature without the $\beta$-independent and $\mu$-independent infinities that plague the continuum path integral formulation.
\end{abstract}

\section{Introduction}

The path integral approach has been extensively applied to the calculation of thermodynamic properties of quantum field theories[1, 2, 3]. Through this approach, the leading contribution to the effective potential of these theories has been calculated in the high temperature limit $\left(\beta \ll 1\right.$, where $\left.\beta=\frac{1}{k T}\right)[2,4]$.

We also have a standard high temperature perturbation theory derived from the path integral expression of the partition function[5,6]. In the calculation of the Helmholtz free energy, via the path integral approach, we do not keep track of contributions that are $\beta$-independent. In general these contributions are irrelevant to the thermodynamic properties of the model, but even though we are not going to deal here with supersymmetric models, we remember that these contributions are crucial to verifying if the supersymmetry is broken or not at finite temperature[7, 8, 9], since for a theory to be supersymmetric the value of its vacuum energy must be zero.

For theories involving bosons, in the path integral we first integrate over the conjugate momenta.
Bernard[1] showed that in this case we have to be careful in constructing the path integral to get the overall $\beta$-dependent constant of the grand canonical partition function. This constant does not appear in pure fermionic models since in these cases the momentum conjugate to a fermionic field is its own hermitian conjugate. Even for these fermionic models, along the calculation of the partition function done via the path integral approach, we get $\beta$-independent terms that are dropped out (see the free fermion case that is fully discussed in reference [3]).

The partition function of free fermion models is calculated directly from its path integral expression, since it is equal to the determinant of its dynamical operator $[3,10]$. When the lagrangean density of the model has self-interacting fermionic terms, it is not possible any more to calculate exactly the non-gaussian noncommutative path integral. One common approach is to get the bosonized version of the model[11]. Another standard way to handle the path integral over non-commutative functions is developing a perturbation theory taking care of the signs coming from fermion loops $[1,2,3]$. Besides the standard perturbation the- 
ory we have the lattice theory that is a non-perturbative approximation approach. Such approach has been very important in the study of QCD (for a review on the subject, see reference [10]). In the lattice formulation of a continuous model at finite temperature, the path integral of the partition function is replaced by a finite space and temperature lattice. Lattice theories have two distinct limits: $i)$ the temperature lattice parameter $\epsilon$ goes to zero $(\epsilon \rightarrow 0)$ to get the path integral expression of the partition function; $i i)$ the space distance $a$ between the nearest sites in each direction goes to zero $(a \rightarrow 0)$ to recover the continuum limit of the model. For fermions on the lattice the naive formulation gives the usual "fermion doubling problem". To get rid of this problem, there are prescriptions in the literature concerning modifications of the Dirac operator on the lattice in such a way that these modifications disappear in the continuous limit[10]. It has been an important test for these prescriptions to be applied to the free fermion model[12]. Not all symmetries of the continuous model are preserved in the lattice prescription; for example, the Wilson action for the free fermion on the lattice[13] breaks the chiral symmetry[10]. Recently, Lüscher[14] showed that this symmetry is exact for a lattice fermion action where the fermion fields satisfy the GinspargWilson identity[15]. Many authors[16, 17, 18] showed that the chemical potential can not be introduced in a naive way in the fermionic lattice model, since even for the free density energy it gives a divergent term proportional to $\left(\frac{\mu}{a}\right)^{2}$. From the previous discussion, we see that the study of free fermion on the lattice has been an important lab to test the prescriptions of the lattice formulation of models involving fermions.

The interesting properties of the non-commutative Grassmann algebra has been applied to get the contributions from spin configurations to the partition function of the classical bidimensional Ising model[19]. In reference [20] Charret et al. proposed a new way to calculate the coefficients of the high temperature expansion of the grand canonical partition function of selfinteracting fermionic models in $d$-dimensions $(d \geq 1)$. They applied the method to the Hatsugay-Kohmoto model[20], that is an exactly solvable model. The approach was also applied to the unidimensional Generalized Hubbard model to get the coefficents up to or$\operatorname{der} \beta^{3}$ of the high temperature expansion of its grand canonical partition function[21]. Differently from other approaches, these coefficients yielded by this method are analytical and exact.

Up to now the approach of Charret et al. has only been applied to fermionic models already regularized on a lattice with space unit one[20, 21]. The aim of the present paper is to use this method to calculate the Helmholtz free energy of the free fermion Dirac, that is, a continuous theory whose exact result is already known in the literature[3]. It is also important to check its application to fermionic lattice models[10].

In section 2 we summarize the results of the method of Charret et al. fully described in reference [20]. In section 3 we apply that approach to the free Dirac fermion using two expansions: in subsection 3.1 we first expand the fermionic field operators in the basis of the energy eigenstates and in subsection 3.2 we consider the naive fermion model on a three dimensional space lattice. In both ways, we show that it is possible to re-sum the high temperature expansion of the grand canonical partition function of the model and compare our results to the ones known in the literature[3, 12]. Certainly, the free Dirac fermion is a good test to the method of Charret et al. In appendix A we present the formulae related to the lagrangean density of the free Dirac fermion. In appendix B we have a "dictionary" of some formulae showing their continuum and discrete expressions.

\section{A Survey of the Approach of Charret et al.}

The grand canonical partition function of any quantum system can be written as a trace over all quantum physical states[3, 22]

$$
\mathcal{Z}(\beta)=\operatorname{Tr}\left[e^{-\beta \mathbf{K}}\right]
$$

where $\beta=\frac{1}{k T}, k$ is the Boltzmann's constant and $T$ is the absolute temperature. The operator $\mathbf{K}$ is defined as $\mathbf{K}=\mathbf{H}-\mu \mathbf{N}, \mathbf{H}$ being the hamiltonian of the system, $\mu$ is the chemical potential and $\mathbf{N}$ is a conserved operator. In the high temperature limit $(\beta \ll 1), \mathcal{Z}(\beta)$ has the expansion

$$
\mathcal{Z}(\beta)=\operatorname{Tr}[1]+\sum_{n=1}^{\infty} \frac{(-\beta)^{n}}{n !} \operatorname{Tr}\left[\mathbf{K}^{n}\right] .
$$

The expectation value of any fermionic operator can be written as a multivariable integral over Grassmann variables[23, 24]. The mapping among the fermionic operators that appear in the fermionic model and the grassmannian generators is such that they satisfy the same algebra. Let $\mathbf{a}_{i}^{\dagger}$ and $\mathbf{a}_{j}$ be the hermitian conjugate fermionic operators that satisfy the anti-commutation relations 


$$
\left\{\mathbf{a}_{i}, \mathbf{a}_{j}^{\dagger}\right\}=\mathbb{1} \delta_{i j} \quad \text { and } \quad\left\{\mathbf{a}_{i}, \mathbf{a}_{j}\right\}=0,
$$

where $i=1, \ldots, \mathcal{N}$. The generators of the associated Grassmann algebra have dimension $2^{2 \mathcal{N}}$, and can be written explicitly as $\left\{\bar{\eta}_{1}, \cdots, \bar{\eta}_{\mathcal{N}} ; \eta_{1}, \cdots, \eta_{\mathcal{N}}\right\}$. They satisfy the following anti-commutation relations:

$$
\left\{\eta_{i}, \eta_{j}\right\}=0, \quad\left\{\bar{\eta}_{i}, \bar{\eta}_{j}\right\}=0 \quad \text { and } \quad\left\{\bar{\eta}_{i}, \eta_{j}\right\}=0
$$

The mapping

$$
\mathbf{a}_{i}^{\dagger} \rightarrow \bar{\eta}_{i} \quad \text { and } \quad \mathbf{a}_{j} \rightarrow \frac{\partial}{\partial \bar{\eta}_{j}},
$$

preserves the algebra (3) due to the fact that the algebra (4) is satisfied by the grassmannian generators.

For any self-interacting fermionic model in a $d$ dimension lattice $(d \geq 1)$, the coefficients of the high temperature expansion (2) can be written as the multivariable Grassmann integral[20]

$$
\begin{aligned}
& \operatorname{Tr}\left[\mathbf{K}^{n}\right]=\int \prod_{I=1}^{2 n N^{d}} d \eta_{I} d \bar{\eta}_{I} e^{\sum_{I, J=1}^{2 n N^{d}} \bar{\eta}_{I} A_{I, J} \eta_{J}} \times \\
& \times \mathcal{K}^{(0)}(\bar{\eta}, \eta, \nu=0) \mathcal{K}^{\circledR(0)}(\bar{\eta}, \eta, \nu=1) \ldots \mathcal{K}^{(0)}(\bar{\eta}, \eta, \nu=n-1),
\end{aligned}
$$

where $N^{d}$ is the number of sites in the $d$-dimensional lattice. We point out that, differently from the lattice version of the path integral, our $d$-dimensional lattice does not include the temperature lattice spacing. Matrix $\mathbf{A}$ is independent of the operators $\mathbf{H}$ and $\mathbf{N}$, and is equal to

$$
\mathbf{A}^{\uparrow \uparrow}=\mathbf{A}^{\downarrow \downarrow}=\left(\begin{array}{ccccc}
\mathbb{1}_{N^{d} \times N^{d}} & -\mathbb{1}_{N^{d} \times N^{d}} & \mathbb{Q}_{N^{d} \times N^{d}} & \ldots & \mathbb{Q}_{N^{d} \times N^{d}} \\
\mathbb{Q}_{N^{d} \times N^{d}} & \mathbb{1}_{N^{d} \times N^{d}} & -\mathbb{1}_{N^{d} \times N^{d}} & \ldots & \mathbb{Q}_{N^{d} \times N^{d}} \\
\vdots & & & & \vdots \\
\mathbb{1}_{N^{d} \times N^{d}} & \mathbb{Q}_{N^{d} \times N^{d}} & \mathbb{Q}_{N^{d} \times N^{d}} & \ldots & \mathbb{1}_{N^{d} \times N^{d}}
\end{array}\right),
$$

where $\mathbb{1}_{N^{d} \times N^{d}}$ and $\mathbb{O}_{N^{d} \times N^{d}}$ are the identity and null matrices of dimension $N^{d} \times N^{d}$, respectively. Due to the fact that the submatrices $\mathbf{A}^{\uparrow \downarrow}$ and $\mathbf{A}^{\downarrow \uparrow}$ are null, the result of the multivariable integral (6) is equal to the product of the independent contributions of the sectors $\sigma \sigma=\Uparrow \uparrow$ and $\sigma \sigma=\downarrow \downarrow$.

The grassmannian function $\mathcal{K}^{(0)}(\bar{\eta}, \eta)$ in eq.(6) is obtained from the normal ordered operator $\mathbf{K}$ by perform-

$$
\mathbf{A}=\left(\begin{array}{cc}
\mathbf{A}^{\uparrow \uparrow} & \mathbb{0} \\
\mathbb{0} & \mathbf{A}^{\downarrow \downarrow}
\end{array}\right)
$$

Each element of $\mathbf{A}$ is, in its turn, a matrix of dimension $n N^{d} \times n N^{d}$, and $(\mathbb{O}$ is the null matrix in that dimension. The indices $I, J$ in matrix A vary in the interval $I, J=1,2, \ldots, 2 n N^{d}$. The matrices $\mathbf{A}^{\uparrow \uparrow}$ and $\mathbf{A}^{\Downarrow \downarrow}$ are equal,

ing the naive mapping: $\mathbf{a}_{i, \sigma}^{\dagger} \rightarrow \bar{\eta}_{I}$ and $\mathbf{a}_{i, \sigma} \rightarrow \eta_{I}$, where $i=1,2, \ldots, N^{d}$ and $\sigma=\uparrow, \downarrow$. The relation between the indices $(i, \sigma)$ and $I$ is given in reference [20].

Finally, the result of the integrals that contribute to $\operatorname{Tr}\left[\mathbf{K}^{n}\right]$ is independent of the $\sigma \sigma$ sector, since $\mathbf{A}^{\uparrow \uparrow}=$ $\mathbf{A}^{\downarrow \downarrow}$. We present here only the results of the integrals of the sector $\sigma \sigma=\uparrow \uparrow$. All those integrals are of the following type: 


$$
M(L, K)=\int \prod_{i=1}^{n N^{d}} d \eta_{i} d \bar{\eta}_{i} \bar{\eta}_{l_{1}} \eta_{k_{1}} \ldots \bar{\eta}_{l_{m}} \eta_{k_{m}} e^{\sum_{i, j=1}^{n N^{d}} \bar{\eta}_{i} A_{i j}^{\dagger \dagger} \eta_{j}},
$$

where $L \equiv\left\{l_{1}, \ldots, l_{m}\right\}$ and $K \equiv\left\{k_{1}, \ldots, k_{m}\right\}$. We should remember that the grassmannians functions $\mathcal{K}^{\text {(1) }}$ are polynomials of the generators of the algebra. The products $\eta \bar{\eta}$ are ordered such that $l_{1}<l_{2}<\ldots<l_{m}$ and $k_{1}<k_{2}<\ldots<k_{m}$. The results of the integrals of type (9) are[25]

$$
M(L, K)=(-1)^{\sum_{i=1}^{m}\left(l_{i}+k_{i}\right)} A(L, K),
$$

where $A(L, K)$ is the determinant obtained from matrix $\mathbf{A}^{\uparrow \uparrow}$ by cutting off the lines $l_{1}, l_{2}, \ldots, l_{m}$ and the columns $k_{1}, k_{2}, \ldots, k_{m} . M(L, K)$ is a co-factor of matrix $\mathbf{A}^{\uparrow \uparrow}$. For fixed $N$, result (10) reduces the calculation of the multivariable integrals over anti-commuting variables to the calculation of co-factors of a well defined matrix whose elements are commuting numbers. It is important to point out that eq.(10) is valid for any integer value of $N$.

In general, it should not be easy to calculate the co-factors of matrix $\mathbf{A}^{\sigma \sigma}$, since each of its elements is a matrix of dimension $N^{d} \times N^{d}$. However, due to the block structure of matrix $\mathbf{A}^{\sigma \sigma}$ Charret et al. showed in reference [20] that it is possible to diagonalize $\mathbf{A}^{\sigma \sigma}$ for any value of $n$ and $N$. The results derived by the application of the method of Charret et al. are analytical in $N$, allowing us to take the thermodynamic limit.

\section{Grand Canonical Partition Function for Free Relativistic Fermions}

The lagrangean density of the free Dirac fermion is

$$
\mathcal{L}=\bar{\Psi}(\vec{x}, t)(\not \partial-m) \Psi(\vec{x}, t),
$$

whose fermionic field operators satisfy the anticommutation relations

$$
\left\{\Psi_{\mu}(\vec{x}, t), \Pi_{\nu}\left(\vec{x}^{\prime}, t\right)\right\}=i \delta_{\mu \nu} \delta\left(\vec{x}-\vec{x}^{\prime}\right), \quad \mu, \nu=1,2,3,4
$$

and

$$
\left\{\Psi_{\mu}(\vec{x}, t), \Psi_{\nu}\left(\vec{x}^{\prime}, t\right)\right\}=\left\{\Pi_{\mu}(\vec{x}, t), \Pi_{\nu}\left(\vec{x}^{\prime}, t\right)\right\}=0,
$$

where $\Pi_{\nu}(\vec{x}, t)$ is the canonical momentum of $\Psi_{\nu}(\vec{x}, t)$ and $\Pi_{\nu}(\vec{x}, t)=i \Psi_{\nu}^{\dagger}(\vec{x}, t)$.

From the lagrangean density (11) and the equation of motion satisfied by the fermionic field operators, the total hamiltonian operator of the system can be written as

$$
\mathbf{H}=\int_{V} d^{3} \vec{x} i \Psi^{\dagger}(\vec{x}, t) \partial_{0} \Psi(\vec{x}, t) .
$$

We use the natural units where $\hbar=c=e=1$. Our metric is $\operatorname{diag}\left(g_{\mu \nu}\right)=(1,-1,-1,-1)$.

\section{III.1 Expansion in the Basis of Energy Eigenstates}

Let us consider a relativistic free fermionic gas at finite temperature contained in a box of volume $V$. The continuous limit is recovered when $V \rightarrow \infty$.

The fermionic operator $\Psi(\vec{x}, t)$ written in the basis of eigenstates of energy of the free Dirac fermion is

$$
\Psi(\vec{x}, t)=\frac{1}{V^{1 / 2}} \sum_{\vec{k}} \sum_{r=1}^{2}\left[\mathbf{a}_{r}(\vec{k}) u_{r}(\vec{k}) e^{-i k_{\mu} x^{\mu}}+\mathbf{b}_{r}^{\dagger}(\vec{k}) v_{r}(\vec{k}) e^{i k_{\mu} x^{\mu}}\right],
$$

where $k_{\mu} x^{\mu}=k_{0} x_{0}-\vec{k} . \vec{x}$, and $k_{0}=\sqrt{|\vec{k}|^{2}+m^{2}}>0$. The destruction fermionic operators $\mathbf{a}_{r}, \mathbf{b}_{r}$ and their respective hermitian conjugates satisfy the anti-commutation relations

$$
\left\{\mathbf{a}_{r}(\vec{k}), \mathbf{a}_{s}^{\dagger}\left(\vec{k}^{\prime}\right)\right\}=\delta_{r s} \delta_{\vec{k}^{,}, \vec{k}^{\prime}} \quad \text { and } \quad\left\{\mathbf{b}_{r}(\vec{k}), \mathbf{b}_{s}^{\dagger}\left(\vec{k}^{\prime}\right)\right\}=\delta_{r s} \delta_{\vec{k}^{\prime}, \vec{k}^{\prime}}
$$


All others anti-commutation relations of these operators are null. The spinor components $u_{r}(\vec{k})$ and $v_{r}(\vec{k})$ are given by eqs.(49) and (50), respectively.

The hamiltonian operator written in terms of creation and destruction operators of the defined energy becomes

$$
\mathbf{H}=-1 \sum_{\vec{k}} \sum_{r=1}^{2} k_{0}+\sum_{\vec{k}} \sum_{r=1}^{2} k_{0}\left[\mathbf{a}_{r}^{\dagger}(\vec{k}) \mathbf{a}_{r}(\vec{k})+\mathbf{b}_{r}^{\dagger}(\vec{k}) \mathbf{b}_{r}(\vec{k})\right],
$$

$\mathbb{1}$ being the identity operator. We define $E_{0}$ as the vacuum energy, $E_{0} \equiv\langle 0|\mathbf{H}| 0\rangle=-2 \sum_{\vec{k}} k_{0},|0\rangle$ being the vacuum state of the fermionic model.

Our aim is to calculate the grand canonical partition function of the free Dirac fermion in contact with some heat and electric charge reservoir. Eqs. (6), (9) and (10) allow us to calculate the coefficients of the high temperature expansion of this function. For the present case, the operator $\mathbf{K}$ in expression (1) is: $\mathbf{K}=: \mathbf{H}:+E_{0} \mathbb{1}-\mu \mathbf{Q}$, where $\mu$ is the chemical potential and $\mathbf{Q}$ is the total electric charge operator of the free relativistic fermions. The operator $\mathbf{Q}$ can be written in terms of the creation and destruction operators as

$$
\begin{aligned}
\mathbf{Q} & =2 \sum_{\vec{k}} \mathbb{1}+\sum_{\vec{k}} \sum_{r=1}^{2}\left[\mathbf{a}_{r}^{\dagger}(\vec{k}) \mathbf{a}_{r}(\vec{k})-\mathbf{b}_{r}^{\dagger}(\vec{k}) \mathbf{b}_{r}(\vec{k})\right] . \\
& \equiv Q_{0} \mathbb{1}+: \mathbf{Q}: .
\end{aligned}
$$

In its turn, the $\mathbf{K}$ operator can be written as

$$
\begin{aligned}
\mathbf{K} & =\sum_{\vec{k}} \sum_{r=1}^{2}\left(k_{0}-\mu\right) \mathbf{a}_{r}^{\dagger}(\vec{k}) \mathbf{a}_{r}(\vec{k})+\sum_{\vec{k}} \sum_{r=1}^{2}\left(k_{0}+\mu\right) \mathbf{b}_{r}^{\dagger}(\vec{k}) \mathbf{b}_{r}(\vec{k})+\mathbb{1}\left(E_{0}-\mu Q_{0}\right) \\
& \equiv \mathbf{K}_{a}+\mathbf{K}_{b}+\mathbb{1}\left(E_{0}-\mu Q_{0}\right) .
\end{aligned}
$$

Due to the anti-commutation relations (16), we have $\left[\mathbf{K}_{a}, \mathbf{K}_{b}\right]=0$, and therefore $\mathcal{Z}(\beta)$ can be written as

$$
\mathcal{Z}(\beta)=e^{-\beta\left(E_{0}-\mu Q_{0}\right)} \mathcal{Z}_{a}(\beta) . \mathcal{Z}_{b}(\beta),
$$

where

$$
\mathcal{Z}_{a}(\beta) \equiv \operatorname{Tr}_{a}\left[e^{-\beta \mathbf{K}_{a}}\right] \quad \text { and } \quad \mathcal{Z}_{b}(\beta) \equiv \operatorname{Tr}_{b}\left[e^{-\beta \mathbf{K}_{b}}\right] .
$$

The calculation of $\mathcal{Z}_{a}(\beta)$ and $\mathcal{Z}_{b}(\beta)$ functions are equivalent. We present here only the details of the calculation of $\mathcal{Z}_{a}(\beta)$. For a free Dirac fermion, the traces for $r=1$ and $r=2$ are equal. Then,

$$
\begin{aligned}
\mathcal{Z}_{a}(\beta) & =\operatorname{Tr}_{a}\left[e^{-\beta \mathbf{K}_{a}}\right]=\left[\operatorname{Tr}_{a}^{(1)}\left[e^{-\beta \mathbf{K}_{a}^{(1)}}\right]\right]^{2} \\
& =\left[\prod_{\vec{k}} \operatorname{Tr}_{a}^{(1)}\left[e^{-\beta\left(k_{0}-\mu\right) \mathbf{n}_{a}^{(1)}(\vec{k})}\right]\right]^{2} .
\end{aligned}
$$

In $\operatorname{Tr}_{a}^{(1)}$ the vector $\vec{k}$ is kept fixed. Since $\mathbf{n}_{a}^{(1)}(\vec{k})$ is a commuting operator, we apply the Newton multinomial expansion formula to write

$$
\operatorname{Tr}_{a}^{(1)}\left[e^{-\beta\left(k_{0}-\mu\right) \mathbf{n}_{a}^{(1)}(\vec{k})}\right]=\operatorname{Tr}_{a}^{(1)}[1]+\sum_{n=1}^{\infty} \frac{(-\beta)^{n}}{n !}\left(k_{0}-\mu\right)^{n} \operatorname{Tr}_{a}^{(1)}\left[\mathbf{n}_{a}^{(1)}(\vec{k})^{n}\right] .
$$

We should note that for fixed $\vec{k}$, the anti-commutation relations (16) are identical to the relations (3); therefore, $\operatorname{Tr}_{a}^{(1)}\left[\mathbf{n}_{a}^{(1)}(\vec{k})^{n}\right]$ can be written as a Grassmann multivariable integral (6) with an equivalent mapping to eq.(5) for the associated generators of the non-commutative algebra. The traces that contribute to eq.(23) are written as the following Grassmann integrals:

$$
\operatorname{Tr}_{a}^{(1)}[\mathbb{1}]=\int \prod_{I=1}^{n} d \eta_{\vec{k}}(I) d \bar{\eta}_{\vec{k}}(I) e^{\sum_{I, I=1}^{n} \bar{\eta}_{\vec{k}}(I) A_{I J}^{(11)} \eta_{\vec{k}}(J)}
$$

and 


$$
\begin{aligned}
\operatorname{Tr}_{a}^{(1)}\left[\mathbf{n}_{a}^{(1)}(\vec{k})^{n}\right] & =\int \prod_{I=1}^{n} d \eta_{\vec{k}}(I) d \bar{\eta}_{\vec{k}}(I) e^{\sum_{I, J=1}^{n} \bar{\eta}_{\vec{k}}(I) A_{I J}^{(11)} \eta_{\vec{k}}(J)} \times \\
\times & \bar{\eta}_{\vec{k}}(1) \eta_{\vec{k}}(1) \ldots \bar{\eta}_{\vec{k}}(n) \eta_{\vec{k}}(n), \quad \text { for }(n>0) .
\end{aligned}
$$

The grassmannian expression for $\operatorname{Tr}_{a}^{(1)}\left[\mathbf{n}_{a}^{(1)}(\vec{k})^{n}\right]$ is obtained from eq.(6) with $N=1$ and $d=1$. Therefore the elements of matrix $\mathbf{A}^{(11)}$ in eq.(8) are just numbers. From eqs.(9) and (10), we conclude that having the $\bar{\eta}$ 's in the integrand corresponds to deleting the first $n$ lines in matrix $\mathbf{A}^{(11)}$ and, in the same way, having the $\eta$ 's corresponds to deleting the first $n$ columns of the same matrix. Therefore, from the expression of matrix $\mathbf{A}^{(11)}$ (see eq.(8)), for arbitrary $n$, we realize that the matrix $\mathbf{A}_{n}^{(11)}$ obtained after cutting off the $n$-first lines and $n$-first columns is an upper triangular matrix whose determinant is equal to 1 . Besides, we have $\operatorname{det}\left[\mathbf{A}^{(11)}\right]=2$, for any value of $n$. In summary, for arbitrary $n$,

$$
\operatorname{Tr}_{a}^{(1)}[1]=2 \quad \text { and } \quad \operatorname{Tr}_{a}^{(1)}\left[\mathbf{n}_{a}^{(1)}(\vec{k})^{n}\right]=1 .
$$

Substituting (26) into expression (25) and resumming it, we get

$$
\operatorname{Tr}_{a}^{(1)}\left[e^{-\beta \mathbf{K}_{a}^{(1)}}\right]=\prod_{\vec{k}}\left(1+e^{-\beta\left(k_{0}-\mu\right)}\right),
$$

that, returning to eq.(20), gives

$$
\mathcal{Z}(\beta)=\left[\prod_{\vec{k}} e^{2 \beta\left(k_{0}+\mu\right)}\left(1+e^{-\beta\left(k_{0}-\mu\right)}\right)\left(1+e^{-\beta\left(k_{0}+\mu\right)}\right)\right]^{2} .
$$

The relation between the Helmholtz free energy and the grand canonical partition function is[22]

$$
\mathcal{W}(\beta)=-\frac{1}{\beta} \ln (\mathcal{Z}(\beta))
$$

Substituting result (28) in eq.(29), the expression derived for the Helmholtz free energy is

$$
\mathcal{W}(\beta)=-\frac{2}{\beta} \sum_{\vec{k}}\left[\beta\left(k_{0}+\mu\right)+\ln \left(1+e^{-\beta\left(k_{0}-\mu\right)}\right)+\ln \left(1+e^{-\beta\left(k_{0}+\mu\right)}\right)\right],
$$

that is equal to the result derived via the continuum path integral formulation of the free Dirac model[3]. Even though our calculation is done in a box of volume $V$, the contribution of the chemical potential derived from expression (30) to the energy density in the zero temperature limit is proportional to $\mu^{4}$ for the massless free fermion model. We remember that the lattice action of the massless free field gives a quadratic divergence if the chemical potencial is introduced in a naive way $[16,17,18]$. For the massless free fermion model, we also get from eq. (30) that the chiral symmetry is exact in this formulation, since $\langle\bar{\Psi} \Psi\rangle=0$ for the fermion condensate.

\section{2 Free Dirac Fermions on the Lattice}

The lattice calculation of models including fermions has been an important tool in learning the properties of these models. The aim of this subsection is to show that the method of Charret et al. can be equally well applied to the lattice version of fermionic models. To do so, we consider the most naive lattice realization of the free Dirac fermion[10]. We remember that the crucial point to apply the method of Charret et al. is working with fermionic field operators that satisfy the anti-commutation relations (3).

The hamiltonian operator of the free Dirac fermion is

$$
\mathbf{H}=\int_{V} d^{3} \vec{x} i \Psi^{\dagger}(\vec{x}, t)\left[\gamma^{0} \vec{\gamma} \cdot \vec{\nabla}-m \gamma^{0}\right] \Psi(\vec{x}, t)
$$

where $\vec{\gamma}=\left(\gamma^{1}, \gamma^{2}, \gamma^{3}\right)$. From appendix B, we get that the operator $\mathbf{H}$ written on the lattice becomes 


$$
\begin{aligned}
\mathbf{H}=\sum_{n_{1}, n_{2}, n_{3}=-\frac{N}{2}}^{\frac{N}{2}} \sum_{\alpha, \beta=1}^{4} & \sum_{j=1}^{3}\left\{i \frac{a^{2}}{2} \Psi_{\alpha}^{\dagger}(\vec{n} a, t)\left(\gamma^{0} \gamma^{j}\right)_{\alpha \beta}\left[\Psi_{\beta}(\vec{n} a+\hat{\jmath} a, t)-\Psi_{\beta}(\vec{n} a-\hat{\jmath} a, t)\right]-\right. \\
& \left.-i a^{3} m \Psi_{\alpha}^{\dagger}(\vec{n} a, t)\left(\gamma^{0}\right)_{\alpha \beta} \Psi_{\beta}(\vec{n} a, t)\right\}
\end{aligned}
$$

where $a$ is the distance between the nearest sites in each space direction. The space point $\vec{x}$ on the lattice is written as $\vec{x}=\vec{n} a . N$ is the total number of space sites in each direction. For simplicity, we take $N$ to be even, although the method applies equally well when $N$ is odd. We only have to be more careful in defining the limits of the sums.

Operator $\mathbf{K}$ in expression (1) is: $\mathbf{K}=\mathbf{H}-\mu \mathbf{Q}$, where $\mu$ is the chemical potential and $\mathbf{Q}$ is the total electric charge operator. The discrete expression of $\mathbf{Q}$ is

$$
\mathrm{Q}=a^{3} \sum_{n_{1}, n_{2}, n_{3}=-\frac{N}{2}}^{\frac{N}{2}} \Psi_{\alpha}^{\dagger}(\vec{n} a, t) \Psi_{\alpha}(\vec{n} a, t)
$$

$$
\left\{\tilde{\psi}_{\alpha}\left(\frac{\pi \vec{l}}{L}, t\right), \tilde{\psi}_{\beta}^{\dagger}\left(\frac{\pi \vec{k}}{L}, t\right)\right\}=\delta_{\alpha \beta} \delta_{r, \vec{k}}^{(3)}
$$

that are identical to relations (3), the necessary algebra to apply the method of Charret et al.

In momentum space, operator $\mathbf{K}$ is written as

$$
\mathbf{K} \equiv \frac{1}{a} \sum_{l_{1}, l_{2}, l_{3}=-\frac{N}{2}}^{\frac{N}{2}-1} \mathbf{K}_{\vec{l}},
$$

where

$$
\mathbf{K}_{\vec{l}} \equiv \tilde{\Psi}^{\dagger}\left(\frac{\pi}{L} \vec{l}, t\right) \mathbf{R}\left(\frac{\pi}{L} \vec{l}\right) \tilde{\Psi}\left(\frac{\pi}{L} \vec{l}, t\right)
$$

and

$$
\tilde{\Psi}\left(\frac{\pi}{L} \vec{l}, t\right) \equiv\left(\begin{array}{c}
\tilde{\psi}_{1} \\
\tilde{\psi}_{2} \\
\tilde{\psi}_{3} \\
\tilde{\psi}_{4}
\end{array}\right)
$$

Imposing periodic spatial boundary conditions on the fermionic field operators, their Fourier decompositions are

$$
\Psi_{\alpha}(\vec{n} a, t)=\frac{1}{\sqrt{V}} \sum_{k_{1}, k_{2}, k_{3}=-\frac{N}{2}}^{\frac{N}{2}-1} \tilde{\psi}_{\alpha}\left(\frac{\pi \vec{k}}{L}, t\right) e^{i \frac{\pi}{L} \vec{k} \cdot \vec{n} a},
$$

where $\alpha=1,2,3,4$ and $L=\frac{N a}{2}$. The fermionic field operators $\Psi_{\alpha}(\vec{x}, t)$ and $\Psi_{\alpha}^{\dagger}(\vec{x}, t)$ satisfy the anticommutation relations (12) and (13), implying that the Fourier components of the fermionic field operators must obey the relations:

and $\quad\left\{\tilde{\psi}_{\alpha}\left(\frac{\pi \vec{l}}{L}, t\right), \tilde{\psi}_{\beta}\left(\frac{\pi \vec{k}}{L}, t\right)\right\}=0$,

The matrix $\mathbf{R}\left(\frac{\pi}{L} \vec{l}\right)$ is defined as

$$
\mathbf{R}\left(\frac{\pi}{L} \vec{l}\right)=\left(\begin{array}{cc}
a(m-\mu) \mathbb{1} & \sin \left(\frac{\pi}{L} l_{j} a\right) \sigma_{j} \\
\sin \left(\frac{\pi}{L} l_{j} a\right) \sigma_{j} & -a(m+\mu) \mathbb{1}
\end{array}\right) .
$$

We have an implicit sum over $j$ in the off-diagonal elements of matrix $\mathbf{R}$ and $\mathbb{1}$ is the identity matrix of dimension $2 \times 2$. Due to the anti-commutation relations (35), we have $\left[\mathbf{K}_{\vec{l}}, \mathbf{K}_{\vec{k}}\right]=0$. Therefore, the grand canonical partition function of the model becomes

$$
\mathcal{Z}(\beta)=\prod_{l_{1}, l_{2}, l_{3}=-\frac{N}{2}}^{\frac{N}{2}-1} \operatorname{Tr}_{\vec{l}}\left[e^{-\frac{\beta}{a} \mathbf{K}_{\vec{\imath}}},\right.
$$

where $T r_{\vec{l}}$ means that the trace is calculated for fixed $\vec{l}$. We should notice that the operator $\mathbf{K}_{\vec{l}}$ is not diagonal 
in momentum space. We perform the similarity transformation $\mathbf{P} \mathbf{R} \mathbf{P}^{-1}=\mathbf{D}$, where the diagonal matrix $\mathbf{D}$ is

$$
\mathbf{D}=\left(\begin{array}{cc}
\lambda_{+} \mathbb{1} & 0 \\
0 & \lambda_{-} \mathbb{1}
\end{array}\right)
$$

and $\lambda_{+}$and $\lambda_{-}$are the eigenvalues of matrix $\mathbf{R}$, namely,

$$
\lambda_{ \pm}=-a(\mu \pm \tilde{\omega}(\vec{l}))
$$

so that

$$
\tilde{\omega}(\vec{l}) \equiv \sqrt{m^{2}+{\tilde{p_{1}}}^{2}+{\tilde{p_{2}}}^{2}+{\tilde{p_{3}}}^{2}},
$$

where $\vec{l} \equiv\left(l_{1}, l_{2}, l_{3}\right)$ and the of variables $\tilde{p}_{i}$ are defined as

$$
\tilde{p}_{i}=\frac{1}{a} \sin \left(p_{i} a\right), \quad i=1,2,3,
$$

with $p_{i}=\frac{\pi}{L} l_{i}$. The new fermionic field operators $\Psi^{\prime}=\mathbf{P} \tilde{\Psi}$ and $\Psi^{\prime \dagger}=\tilde{\Psi}^{\dagger} \mathbf{P}^{-1}$ also preserve the anticommutation relations (35).

The function $\mathcal{Z}(\beta)$ written in terms of the new fermionic field operators has the same form as the r.h.s. of eq.(22). Following similar steps, we get the Helmholtz free energy for the model on the lattice, that is,

$$
\begin{aligned}
\mathcal{W}(\beta) & =-2 \sum_{l_{1}, l_{2}, l_{3}=-\frac{N}{2}}^{\frac{N}{2}-1}(\mu+\tilde{\omega}(\vec{l}))- \\
-\frac{2}{\beta} & \sum_{l_{1}, l_{2}, l_{3}=-\frac{N}{2}}^{\frac{N}{2}-1}\left[\ln \left(1+e^{-\beta(\tilde{\omega}(\vec{l})+\mu)}\right)+\ln \left(1+e^{-\beta(\tilde{\omega}(\vec{l})-\mu)}\right)\right] .
\end{aligned}
$$

In the limit $a \rightarrow 0$ the function $\mathcal{W}(\beta)$ agrees with eq.(2.4) of reference [12], after summing over Matsubara frequencies, being equal to twice the result (30). Here, as usual in lattice calculations with fermions, the doubling problem is lifted by including the Wilson term

$$
\mathbf{H}^{(W)}=i \frac{r a}{2} \int_{V} d^{3} \vec{x} \Psi^{\dagger}(\vec{x}, t) \gamma^{0} \nabla^{2} \Psi(\vec{x}, t),
$$

in hamiltonian (31), where $r$ is Wilson's constant.

For models that are quadratic in the fermionic fields, the result of the partition function is the same for any value of the temperature parameter $\epsilon\left(\epsilon=\frac{\beta}{N}\right)$ [26]. That is why our result (45) can be directly compared to the one derived by the lattice calculation of hamiltonian (32), even the Wilson term (46) is added to it, since both expressions already give the exact results in the respective method.

\section{Conclusions}

Recently, Charret et al. proposed a new way to calculate the coefficients of the high temperature expansion of the grand canonical partition function $\mathcal{Z}(\beta)$ of any self-interacting fermionic model in $d$-dimensions $(d \geq 1)[20]$. In order to apply this method, it is enough to write the second quantized expression of the hamiltonian and a given conserved operator in terms of operators satisfying the anti-commutation relations (3). In this approach, at each order $\beta^{n}$ of the high temperature expansion of the function $\mathcal{Z}(\beta)$, the calculation of the coefficients is reduced to obtaining the co-factors of a matrix with commuting entries - see matrix $\mathbf{A}$ in eqs. (7) and (8). For a fixed number $N$ of sites on the lattice, all the mathematical objects in the calculation are well-defined. The value of $N$ can be even or odd. This approach works with grassmannian functions, but it avoids calculating the fermionic path integral of $\mathcal{Z}(\beta)$.

We applied the method of Charret et al. to the free Dirac fermion by first expanding it in the basis of the eigenstates of energy of the free fermions. The hamiltonian and the total electric charge operators (eqs.(14) and (18) respectively) include their respective vacuum contributions. Result (30) gives two divergent terms to the Helmholtz free energy $\mathcal{W}(\beta)$ : the vacuum energy and the electric charge of the vacuum. This last divergent term does not appear when we perform calculations via the usual path integral approach[3], while 
the $\beta$-dependent terms in $\mathcal{W}(\beta)$ are identical in both methods. In the path integral calculation[3], divergent terms that are $\beta$ and $\mu$-independent are dropped out. This does not happen in the present approach. The calculation is carried out on a lattice model in the momentum space, but the contribution of the chemical potential to the energy density of massless free fermions in the zero temperature limit is proportional to $\mu^{4}$ - as it should be, since the continuum limit result is recovered in this method. This approach also preserves the chiral symmetry of the continuous massless Dirac fermion.

Since the calculation of fermionic lattice models is an important tool, we also applied the approach of Charret et al. to a naive version of the free Dirac fermion on the lattice[10]. In this case we also get the usual doubling problem as in fermion lattice calculations; it can be lifted by the Wilson term. The direct agreement between our results and the lattice calculations comes from the fact that we are considering quadratic fermionic models and therefore we do not need to take $\epsilon \rightarrow 0$ in the lattice to get the correct results.

In summary, we can affirm that the method of Charretet al. can also be applied to continuous fermionic models. It is an analytical approach that allows us to calculate all terms of the function $\mathcal{Z}(\beta)$ for the free
Dirac fermion without ambiguity, including the divergent terms coming from the vacuum contribution. The momentum space lattice that we employed to perform our calculations for the free Dirac fermion model gives the correct continuum limit and preserves the quantum symmetries of the model. This could be an indication that it is a candidate for the fermionic lattice action of interacting fermionic fields that should be calculated using the method of Charret et al.[20]. The next step in this approach is that of studying the renormalization scheme associated to physical quantities for fermionic models with self-interacting terms.

\section{Acknowledgements}

The authors thank M. Creutz for comments and pointing out that in the present approach the chemical potential problem is naturally solved, and for bringing reference [16] to our attention. O.R.S. and M.T.T. are in debt with $H$. Rothe for valuable discussions about lattice fermionic free models, and for reading and making suggestions to the original version of the paper. O.R.S. thanks CAPES for total financial support. S.M.de S. and M.T.T. thank CNPq for partial financial support. M.T.T. also thanks FINEP and S.M.de S. thanks FAPEMIG for partial financial support.

\section{Appendix}

\section{A Useful Formulae}

In the lagrangean density for the free Dirac fermion (see eq.(11)),

$$
\mathcal{L}=\bar{\Psi}(\vec{x}, t)(\not \partial-m) \Psi(\vec{x}, t)
$$

the Dirac matrices $\gamma^{\mu}, \mu=0, . ., 3$, are:

$$
\gamma^{0}=i\left(\begin{array}{cc}
1 & 0 \\
\mathbb{0} & -1
\end{array}\right) \quad \text { and } \quad \gamma^{i}=i\left(\begin{array}{cc}
0 & \sigma_{i} \\
-\sigma_{i} & 0
\end{array}\right), \quad i=1,2,3 \text {. }
$$

The matrices $\sigma_{i}, i=1,2,3$ are the Pauli matrices, and $\mathbb{1}$ and $(\Phi$ are the identity and null matrices of dimension $2 \times 2$, respectively. We have $\bar{\Psi}(\vec{x}, t)=-i \Psi_{\nu}^{\dagger}(\vec{x}, t) \gamma_{0}$.

The spinors components $u_{r}(\vec{k})$ and $v_{r}(\vec{k})$ in eq. $(15)$ are

$$
\begin{aligned}
& u_{1}(\vec{k})=\sqrt{\frac{m+k_{0}}{2 k_{0}}}\left(\begin{array}{c}
1 \\
0 \\
\frac{k_{3}}{m+k_{0}} \\
\frac{k_{1}+i k_{2}}{m+k_{0}}
\end{array}\right), \quad u_{2}(\vec{k})=\sqrt{\frac{m+k_{0}}{2 k_{0}}}\left(\begin{array}{c}
0 \\
1 \\
\frac{k_{1}-i k_{2}}{m+k_{0}} \\
\frac{-k_{3}}{m+k_{0}}
\end{array}\right), \\
& v_{1}(\vec{k})=\sqrt{\frac{m+k_{0}}{2 k_{0}}}\left(\begin{array}{c}
\frac{k_{1}-i k_{2}}{m+k_{0}} \\
\frac{-k_{3}}{m+k_{0}} \\
0 \\
1
\end{array}\right), \\
& v_{2}(\vec{k})=\sqrt{\frac{m+k_{0}}{2 k_{0}}}\left(\begin{array}{c}
\frac{k_{3}}{m+k_{0}} \\
\frac{k_{1}+i k_{2}}{m+k_{0}} \\
1 \\
0
\end{array}\right) .
\end{aligned}
$$




\section{B Summary of Expressions on the Lattice}

We assume that each direction in three dimensional space has $N$ sites. For simplicity, $N$ is assumed to be even. The distance between nearest points along each direction is $a$. In the space lattice, each point is written as $\vec{x}=\vec{n} a$, where $\vec{n}=\left(n_{1}, n_{2}, n_{3}\right), n_{i}=-\frac{N}{2},-\frac{N}{2}+1, \cdots, \frac{N}{2}$, and $i=1,2,3$.

We have the following "dictionary" between the continuous and discrete representations of the formulae:

$$
\begin{aligned}
\int_{V} d^{3} \vec{x} & \rightarrow a^{3} \sum_{n_{1}, n_{2}, n_{3}=-\frac{N}{2}}^{\frac{N}{2}} \\
\delta\left(\vec{x}-\vec{x}^{\prime}\right) & \rightarrow \frac{1}{a^{3}} \delta_{\vec{n}, \vec{m}}^{(3)} \equiv \frac{1}{a^{3}} \delta_{n_{1}, m_{1}} \delta_{n_{2}, m_{2}} \delta_{n_{3}, m_{3}},
\end{aligned}
$$

where $\vec{x}=\vec{n} a, \vec{x}^{\prime}=\vec{m} a$ with $\vec{n}=\left(n_{1}, n_{2}, n_{3}\right)$ and $\vec{m}=\left(m_{1}, m_{2}, m_{3}\right)$, respectively.

Finally, the first derivative defined on the lattice is defined as[10]

$$
\partial_{i} \Psi_{\alpha}(\vec{x}, t) \rightarrow \frac{1}{2 a}\left[\Psi_{\alpha}(\vec{n} a+\hat{\imath} a, t)-\Psi_{\alpha}(\vec{n} a-\hat{\imath} a, t)\right],
$$

where $\hat{\imath}$ is the unit vector in the direction $i$.

\section{References}

[1] C.W. Bernard, Phys. Rev. D 9, 3312 (1974).

[2] L. Dolan and R. Jackiw, Phys. Rev. D 9, 3320 (1974).

[3] J. I. Kapusta, Finite Temperature Field Teory, Cambridge University Press (1989), cap. 2.

[4] Y. İpekoğlu, M. Leblanc and M.T. Thomaz, Ann. of Phys. 214, 160 (1992).

[5] J.A. Henderson, J. Oitmaa and M.C.B. Ashey, Phys. Rev. B 46, 6328 (1992-II).

[6] R.R.P. Singh and R.L. Glenister, Phys. Rev. B 46, 11871 (1992-II).

[7] A. Das, Phys. A 158, 1 (1989).

[8] A. Mishra, H. Mishra, S.P. Misra and S.N. Nayak, Phys. Lett. B 251, 541 (1990).

[9] S. Kumar, J. Phys. A 23, L127 (1990).

[10] H. Rothe, Lattice Gauge Theories - An Introduction, World Scientific (1996) and references therein.

[11] S. Coleman, Phys. Rev. D 11, 2088 (1975).

[12] I. Bender, H.J. Rothe, I.O. Stamatescu and W. Wetzel, Z. Physik C 58, 333 (1993).

[13] K.G. Wilson, New Phenomena in Subnuclear Physics (Erice, 1975), ed. A. Zichichi (New York, Plenum, 1975).

[14] M. Lüscher, Phys. Lett. B 428, 342 (1998).

[15] P.H. Ginsparg and K.G. Wilson, Phys. Rev. D 25, 2649 (1982).
[16] P. Hasenfratz and F. Karsch, Phys. Lett. B 125, 308 (1983).

[17] J. Kogut, H. Matsuoka, S. Shenker, J. Shigemitsu, D.K. Sinclair and H.W. Wyld, Nucl. Phys. B 225, 93 (1983).

[18] R.V. Gavai, Phys. Rev. D 32, 519 (1985).

[19] V. N. Plechko, Sov. Phys. Dokl. 30, 271 (1985), V. N. Plechko, JINR. Dubna (1986) 748, F. A. Berezin, Usp. Mat. Nauk, 24, 748 (1969).

[20] I. C. Charret, E. V. Corrêa Silva, S. M. de Souza, O. Rojas Santos and M. T. Thomaz, Grassmann Algebra and Fermions at Finite Temperature, J. Math. Phys. (in press).

[21] I. C. Charret, E. V. Corrêa Silva, O. Rojas Santos, S. M. de Souza and M. T. Thomaz, Unidimensional Generalized Hubbard Model in the High Temperature Limit, Physica A (in press).

[22] F. Reif Fundamental of Statistical and Thermal Physics, Mc-Graw-Hill (1965), cap. 9.

[23] C. Itzykson and J. B. Zuber, Quantum Field Theory, Mc. Graw-Hill (1980) cap. 9.

[24] I. C. Charret, E. V. Corrêa Silva, S. M. de Souza, Physica A 264, 204 (1999).

[25] I. C. Charret, S. M. de Souza and M. T. Thomaz, Braz. Journ. of Phys. 26, 720 (1996).

[26] H.J. Rothe, Lattice Fermions at Non-Zero Temperature and Chemical Potential and Divergences in Thermodynamical Observables, pre-print HD-THEP-93-12. 